\title{
Inline viscometery for non-Newtonian viscosity characterization
}

\author{
Miha Završnik, Martin Joseph-Strasser \\ Anton Paar GmbH, Anton-Paar-Str. 20, 8054 Graz, Austria, \\ miha.zavrsnik@anton-paar.com
}

\begin{abstract}
:
Dynamic viscosity is an important parameter for quality control in a wide range of industrial processes and is one of the most important fluid properties for the production of many significant industrial substances. In particular non-Newtonian viscosity behavior of heterogeneous mixtures (i.e., dispersions, suspensions) and polymeric solutions is an important material property that contributes to a fluid's performance, and is often the main source of problems in handling, processing, and application.

The objective of this contribution is to present a novel process viscometer based on the fluid dynamic measurement principle. The measurement principle and construction allow its use under harsh field conditions, providing actual unambiguous (non-Newtonian) viscosity as a function of the shear rate. Representative examples of non-Newtonian viscosity characterization using experimental data on real materials will be presented and good correlation between laboratory and process will be demonstrated.
\end{abstract}

Key words: dynamic viscosity, process, in-line, non-Newtonian behavior

\section{Introduction}

In many industrial processes ranging from chemical and manufacturing industries to medicine and food processing, viscosity is an important parameter for intermediate and final product characterization.

Traditionally one would take samples one by one out of the process and examine them with a laboratory viscometer, typically including a range of shear rates as most of the fluids in industry are non-Newtonian. However, there are some difficulties with the traditional method. For example, viscosity can be directly affected by temperature, shear rate, and other variables that can be very different during laboratory measurement from what they are in-line. Since rheological properties of many samples are closely related to the flow conditions in the process, the measurements in laboratory may lead to inaccurate results [3].

In contrast to laboratory (offline) determination of viscosity, process viscometers gather in-line information on viscosity directly within the process and in real time. They are several different in-line viscometers available, implementing measurement principles ranging from capillary differential pressure, time of falling or oscillating elements, torsional oscillations damping and rotation rate measurement of a solid shape upon application of a known force or torque [1]. According to our knowledge, except for the rotation methods, most of the viscometers lack from a welldefined shear field and/or operate at very high shear rates. Due to that, measurements of nonNewtonian industry fluids which in most cases exhibit pseudoplastic behavior could be very difficult. On the other hand standard rotation methods often allow only a by-pass installation or do not allow for good exchange of fluid in the measuring field.

In this paper we will describe a novel fluid dynamic inline viscometer. It is based on a rugged mechanical configuration with a tapered gap between two bodies applying shear forces to the sample. By doing this, the advantages of the rotation measurement is maintained and at the same time robustness, good sample exchange and the ability to install the 
instrument in-line, in a bypass or tank is supplemented.

\section{Theory and Modeling}

The process viscometer is based on a similar hydrodynamic effect which makes slide bearings work. In such bearings, the relative motion between two surfaces induces shear stress in a lubricant and leads to the formation of a lubricant film separating the sliding surfaces. The pressure necessary to carry the load can only develop if the film is wedgeshaped, so that the variable surface will be slightly inclined. The pressure distribution in the wedge-shaped film depends on the surface velocity and the dynamic viscosity of the lubricant.

For the presented process viscometer this viscosity dependence is utilized to measure the viscosity given a fixed geometrical configuration of the sliding surfaces. The functional principle is shown in Figure 1. A rotor and a static outer surface define the wedge-shaped gap of the viscometer configuration. The rotor can be powered directly from a rigid shaft or using a magnetic coupling. The fluid under test is conveyed into the gap by the action of the rotor. The opposite static outer surface is rigidly fixed on the entry side and open-ended at the outlet.

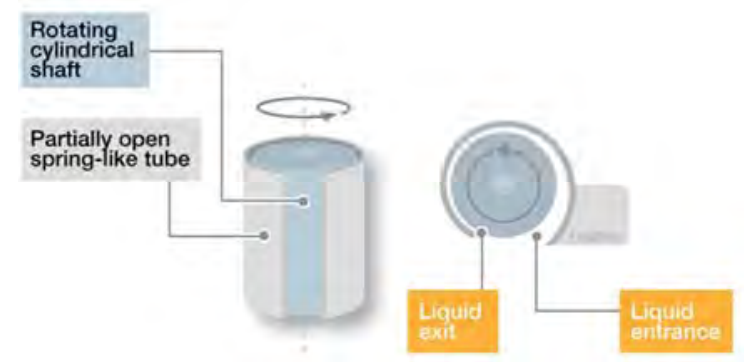

Fig. 1. Partially open tube with a rotating cylindrical shaft

Due to the pressure rise in the gap, the outer surface is slightly displaced, acting as a spring. The displacement between the fixed and flexible parts of the spring element is proportional to the fluids viscosity and measured with an inductive sensor. The sensor coil is mounted on the fixed part of the spring while the counter piece is attached to the flexible part [1, 2].

Liquid surrounding the system is drawn into the narrowing gap by shear forces. The liquid volume passing through the gap is constant and as a consequence the pressure changes. The pressure first increases as the gap gets thither, then reaching a maximum inside the tube, then decreasing again, as it exits. This pressure is a measure of liquids viscosity, being proportional to the dynamic viscosity, shear velocity and a geometry factor:

$$
\begin{aligned}
& p^{\prime}=\frac{d p}{d x}=f\left\{\eta, \omega,\left(\frac{1}{h}\right)^{2}\right\} \\
& \eta-\text { dynamic viscosity } \\
& \begin{array}{l}
\omega-\text { bob rotation speed } \\
h-\text { gap }
\end{array}
\end{aligned}
$$

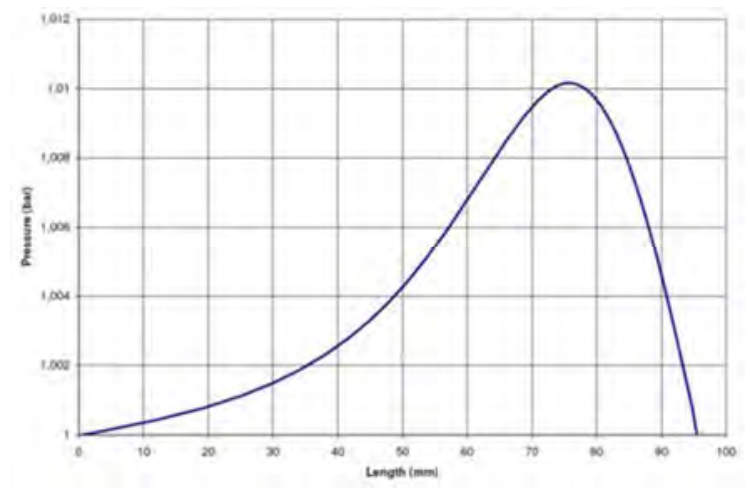

Fig. 2. Approximate pressure distribution along the gap

Besides the mechanical components outlined, the viscometer incorporates an electric drive with closed-loop control to achieve stable flow conditions and shear rate in the wedge-shaped gap. The excess pressure is zero at the inlet and outlet and reaches a maximum after about two thirds of the gap length (Fig. 2). The spring structure leads to a weighted integration of the pressure distribution, which is measured as displacement. An inductive displacement sensor measures the deflection of the spring, offering the advantage of being independent of the process fluid and decoupled from the rotating motion and hence not influenced by bearing friction changes. A temperature sensor immersed in the process liquid is used to compensate for temperature variations of the sensor front-end and to provide process temperature information.

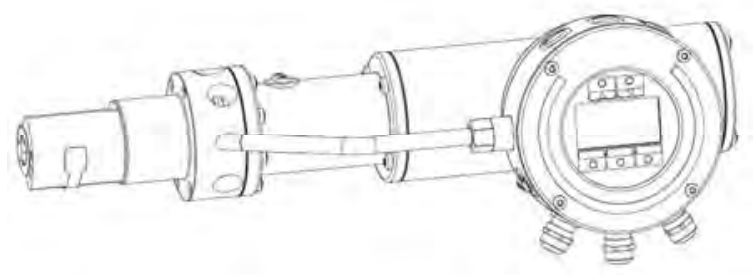

Fig. 3. Dynamic fluid inline viscometer

By design, new sample is continuously drawn into the gap. The transducer signal is acquired with a robust contactless measurement, allowing high pressure and temperature ratings of the instrument. 


\section{Experimental Setup}

Two general cases have been investigated during the experiment. In order to verify basis unit performance, a viscosity evaluation using Newtonian fluids has been performed first. Here standard viscosity oils have been used. The range has been chosen from $1 \mathrm{mPas}$ to approximately $100 \mathrm{mPas}$ and is corresponding to viscosities of typical process polymer solutions within a moderate shear rate range.

Second, multiple shear rate measurements of non-Newtonians have been performed. Here a polyacrylamide polymer solution with shear thinning behavior with two distinctive concentrations has been used.

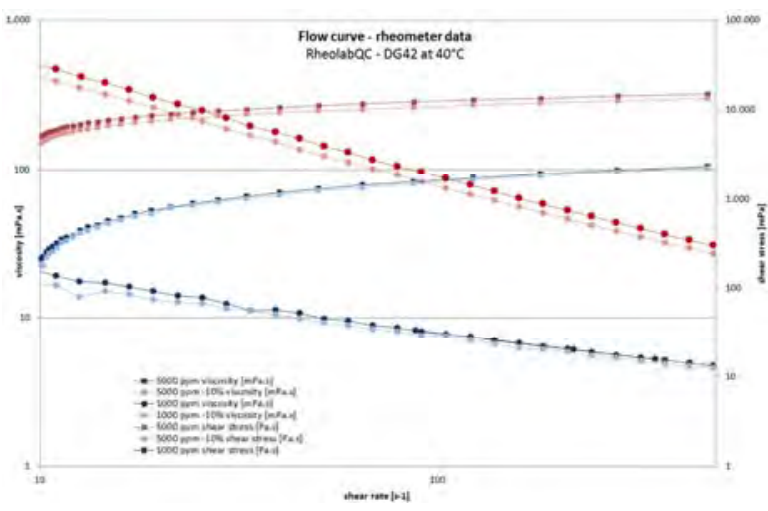

Fig. 4. Polymer solutions rheometer data

The viscosities of polymer solutions were measured using a rheometer with the double gap cylindrical measuring system. Because viscosity is sensitive to temperature, all measurements were performed at constant temperature conditions around $40^{\circ} \mathrm{C}$. Figure 4 shows typical flow curves (viscosity and shear stress) for shear rates between 10 to 500 reciprocal seconds at a constant temperature of $40{ }^{\circ} \mathrm{C}$. The red colored graphs represent a polymer solution of $5000 \mathrm{ppm}$, whereas the blue colored a solution of $1000 \mathrm{ppm}$. Additionally to these 2 types of solution a dilution of $10 \%$ was prepared (light red and blue graphs).

Experiments were carried out by mounting the inline viscometers inside a tank with a double wall. By temperature control of the liquid within the double wall, the temperature within the tank has been controlled. A small stirrer inside the tank allowed for smooth mixing and uniform distribution of the probe inside the tank.

In the first case the tank has been filled with standard viscosity oil, whereas in second case the tank has been filled with the appropriate concentration of the polymer solution. During the experiment data logging of the inline viscometer has been performed using standard fieldbus connection.

\section{Measurements and Results}

Newtonian fluid

After performing several viscosity cycles the graph presented in Figure 5 could be obtained.

Within the investigated viscosity range the following performance factors have been aquired:

Accuracy $<0,5 \mathrm{mPa}$.s

Repeatability $<0,1 \mathrm{mPa}$.s

Linearity $<0,25 \mathrm{mPa}$.s

Hysteresis $<0,1 \mathrm{mPa}$.s 


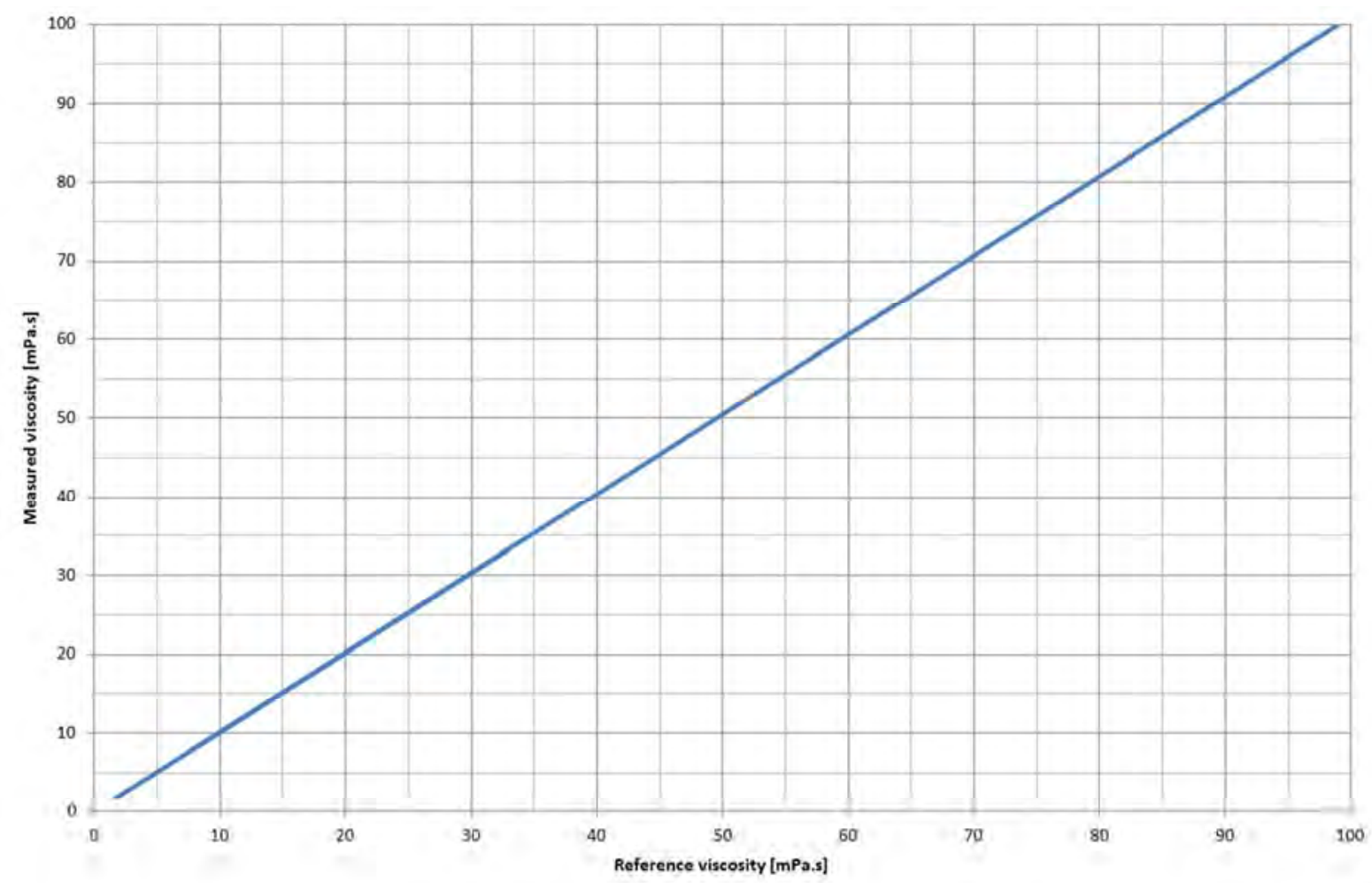

Fig. 5. Inline viscometer - Newtonian characterization

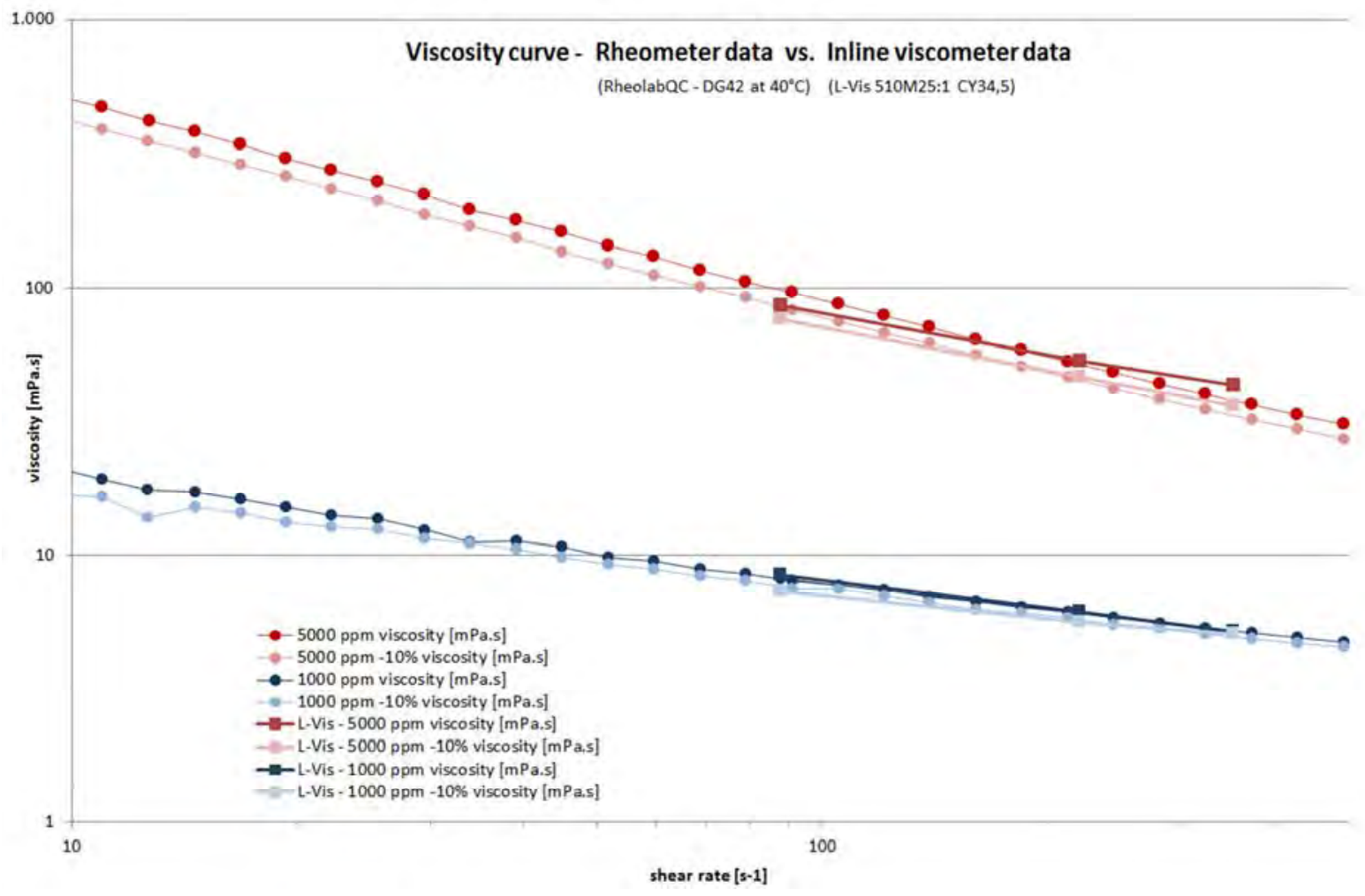

Fig. 6. Viscosity curve - Rheometer versus Inline viscometer 
Non-Newtonian fluid

The presented inline viscometer is designed to be able to measure at 3 different speeds of the measuring cylinder. According to the geometry factor used for the experiment, these speeds correspond to shear rates of 88,220 and 352 reciprocal seconds. During the non-Newtonian measurement cycle the inline viscometer is switching through all three speeds, whereby measuring three apparent viscosity values.

Figure 6 shows the measurement at those 3 shear rates (speeds) for the $5000 \mathrm{ppm}$ polymer solution (red graph) and the $1000 \mathrm{ppm}$ (blue graph). As with the rheometer measurements both polymer solutions were diluted by $10 \%$ (light red and light blue graph). All measurements were done at fairly stable temperature conditions of 40,8 to $40,9^{\circ} \mathrm{C}$.

Comparing the inline viscometer results with the laboratory rheometer characterization, we obtain a clear and unambitious distinction between the diluted and non-diluted polymer concentrations even in the case of the low concentration polymer solution. A distinctive change of approximately $1 \mathrm{mPa}$.s could be detected whereby at the same time the apparent viscosity values maintained the overall shape of the laboratory viscosity curve.

\section{Discussion and Conclusion}

In this paper, we have described a new inline process viscometer allowing for the quantitative characterization of unambiguous (nonNewtonian) viscosity as a function of the shear rate. Using a rugged mechanical configuration with a tapered gap we have been able to decouple the rotating motion of the cylinder from the measurement while providing excellent sample exchange and maintained good accuracy and repeatability.

The new device gives operators the means to better control their process, as well as measure the impact of their corrective actions. They can standardize operations, allowing them to save money and get a uniform product that meets their quality standards.

\section{References}

[1] M. Zavrsnik, Process Viscometers - Chapter 8.64, 5th edition of the Instrument and Automation Engineer's Handbook, CRC Press, 2013

[2] G. Steiner, J. Gautsch, R. Breidler, F. Plank, A novel fluid dynamic inline viscometer suitable for harsh process conditions, Proc. Eurosensors XXIV, September 5-8, 2010

[3] V. Koseli, S. Zeybek, Y. Uludag, Online Viscosity Measurement of Complex Solutions Using
Ultrasound Doppler Velocimetry, Turk J Chem, 297-305, 30, 2006 